\title{
CORRESPONDENCE
}

\section{From BPD to COPD? The hypothesis is intriguing but we lack lung pathology data in humans}

\section{To the Editors:}

We read the study reported by DIDON et al. [1] and the accompanying editorial [2] with great interest because they deal with an emerging area of paediatric and adult respiratory medicine, i.e. the origins in the early life of chronic obstructive pulmonary disease (COPD) and genetic susceptibility to lung damage.

In a rodent model, the study shows that lung-specific epithelial deletion of CCAAT/enhancer binding protein $\alpha(\mathrm{C} / \mathrm{EBP} \alpha)$ disrupts normal lung morphogenesis in fetal life, subsequently driving the onset of spontaneous severe chronic bronchial inflammation, and ultimately results in pulmonary emphysema in adult mice [1].

The authors understandably interpret their observations with reference to bronchopulmonary dysplasia (BPD), the main chronic respiratory complication of prematurity [3]. Indeed, their animal model shows both the well-known alveolar and vascular growth inhibition seen after very premature birth, and the COPD-like changes dreaded as a long-term consequence of BPD.

We believe that some points need to be clarified, however, and warrant further discussion. BPD is the earliest and perhaps longest-lasting obstructive lung disease in humans. Initially described ( $>40$ yrs ago) as a disease characterised by intense bronchial inflammation and massive parenchymal disruption in relatively immature newborns with surfactant deficiency ("old BPD"), BPD has changed strikingly over time. The form of BPD more recently seen ("new BPD") essentially involves neonates born before 30 weeks of gestation and weighing $<1,000 \mathrm{~g}$ at birth, and it is interpreted as a disruption in the normal developmental pathway of the lung, which is exposed to multiple injuries while still in the early stages of its morphogenesis. This leads to its structural simplification with the development of fewer, larger alveoli, a dysmorphic vascular growth and a generally reduced surface available for gas exchange. The airways are spared to some degree and inflammation is usually less prominent than in the old form of BPD [3].

The natural history of BPD is still incompletely understood. There is evidence that BPD survivors reach a reduced maximal airway function (judging from reductions in forced expiratory volume in $1 \mathrm{~s}$ (FEV1) and FEV1/forced vital capacity) as young adults [3], and some even show a trend towards an early and steeper decline in lung function with age [4,5], raising concern that BPD may be a precursor of a COPD-like phenotype later in life.
DIDON et al. [1] speculate in their paper that long-standing BPD and COPD share some underlying mechanisms and similar pathological patterns. We feel, however, that they have overlooked the fact that lung pathology data on BPD patients are only available in the early stages of the disease, i.e. within the first months of life, while virtually nothing is known about the lung pathology of survivors of BPD beyond infancy, so nobody knows how the picture evolves over time.

Some imaging studies have shown emphysematous lung changes in young adults who have survived BPD [6], as DiDON et al. [1] correctly mention. However, the accompanying intense COPD-like inflammation that they saw in their experimental model may not apply to BPD-induced late pulmonary changes. The emphysematous changes in survivors of BPD might be a consequence of ventilator-associated parenchymal injury sustained early in life (in old BPD) [6], or reflect the enlargement of distal airspaces due to incomplete alveolar septation (as in new BPD); a feature reported in the only pathological sample currently available from a patient with long-term BPD [7].

Inflammation certainly has a role in the early stages of BPD but no data currently exist to support the assumption that BPDinduced late pulmonary changes are based on persisting or subsequently developing inflammatory mechanisms, as DIDON et al. [1] speculate.

Before their model can be considered plausible as reflecting the respiratory outcome of $\mathrm{BPD}$, at least some of the pathological changes they found in adult mice with $\mathrm{C} / \mathrm{EBP} \alpha$ deletion (e.g. peribronchial lymphoid infiltrates, bronchiolitis, mucus plugging and macrophage accumulation) should be documented in long-term survivors of BPD. Providing evidence of the inflammatory nature of the late consequences of BPD might support the development of evidence-based therapeutic or preventive measures, enabling us to go beyond the current empirical approach to the treatment of BPD beyond infancy.

Although we acknowledge the importance of the experimental findings reported by DIDON et al. [1] on the matter of the origins of COPD in early life, we believe that the assumption of a link between BPD and COPD can only be placed in the appropriate context after lung pathology data on long-term survivors of BPD have become available.

\section{Filippone, S. Carraro and E. Baraldi}

Dept of Paediatrics, Unit of Respiratory Medicine and Allergy, Unit of Neonatal Intensive Care, University of Padua, School of Medicine, Padova, Italy. 
Correspondence: E. Baraldi, Dept of Paediatrics, Via Giustiniani 3, 35128 Padova, Italy. E-mail: baraldi@pediatria. unipd.it

Statement of Interest: None declared.

\section{REFERENCES}

1 Didon L, Roos AB, Elmberger GP, et al. Lung-specific inactivation of CCAAT/enhancer binding protein $\alpha$ causes a pathological pattern characteristic of COPD. Eur Respir J 2010; 35: 186-197.

2 Shi W, Warburton D. Is COPD in adulthood really so far removed from early development? Eur Respir J 2010; 35: 12-13.

\section{From the authors:}

We would like to thank M. Filippone and co-workers for their comments regarding our paper, "Lung-specific inactivation of CCAAT/enhancer binding protein $\alpha$ causes a pathological pattern characteristic of COPD" [1]. We share their concern regarding the lack of human studies addressing the adult phenotype of former bronchopulmonary dysplasia (BPD) patients, and hope to continue this discussion. M. Filippone and co-workers address the difference between "old" and "new" BPD phenotypes, which warrants further discussion with regards to the findings presented in our paper. Old BPD, characterised by intense bronchial inflammation and massive parenchymal disruption in relatively immature newborns with surfactant deficiency, is greatly believed to be a consequence of oxygen ventilator treatment [2]. In contrast, new BPD, as M. Filippone and co-workers state, involves extremely premature neonates that consequently demonstrate disrupted lung development resulting in immature lungs, vulnerable to multiple injuries. In our publication, when comparing the phenotype of mice with lung-specific inactivation of CCAAT/enhancer binding protein $\alpha\left(\mathrm{Cebpa}^{4 L E}\right)$ presenting BPD pathology, we were referring to new BPD that, like in Cebpa ${ }^{\triangle L E}$ mice, is characterised by immature lung morphology. That is, a structural simplification with fewer, larger alveoli causing reduced surface available for gas exchange; a dysmorphic vascularity; and very limited, if any, inflammation [2].

One prominent difference between adult and neonatal or young Cebpa ${ }^{\triangle L E}$ mice is the development of a spontaneous inflammation as the mice reaches adulthood. The origin of this inflammation remains unknown; however, the spontaneous inflammation in our animals presents an opportunity to study the sensitivity of a lung with a morphological resemblance of new BPD to inflammation. There is a possibility, as mentioned by M. Filippone and co-workers, that the pathology of long-term survivors of new BPD includes an inflammatory component
3 Baraldi E, Filippone M. Chronic lung disease after premature birth N Engl J Med 2007; 357: 1946-1955.

4 Doyle LW, Faber B, Callanan C, et al. Bronchopulmonary dysplasia in very low birth weight subjects and lung function in late adolescence. Pediatrics 2006; 118: 108-113.

5 Filippone M, Bonetto G, Cherubin E, et al. Childhood course of lung function in survivors of bronchopulmonary dysplasia. JAMA 2009; 302: $1418-1420$

6 Wong PM, Lees AN, Louw J, et al. Emphysema in young adult survivors of moderate-to-severe bronchopulmonary dysplasia. Eur Respir J 2008; 32: 321-328.

7 Cutz E, Chiasson D. Chronic lung disease after premature birth N Engl J Med 2008; 358: 743-745.

DOI: $10.1183 / 09031936.00013310$

not triggered by external factors, such as tobacco smoke, which could contribute to the development of an obstructive lung disease. Although, further investigations in long-term survivors of BPD need to be conducted to investigate the presence of an inflammation before such conclusions can be drawn. However, if there is a spontaneous inflammation in long-time survivors of BPD, this might provide a valuable opportunity for treatment, as it might prove beneficial to suppress this inflammation to prevent development of chronic obstructive pulmonary disease. Finally, these findings are from an animal model and, in agreement with M. Filippone and co-workers, we urge further clinical studies to address the link between respiratory problems early in life and respiratory complications, such as chronic obstructive pulmonary disease, later in life.

\section{Didon*, A. Roos ${ }^{\#}$ and M. Nord ${ }^{\#}$}

*Dept of Genetic Medicine, Weill Cornell Medical College, New York, NY, USA. "Dept of Medicine, Div. of Respir Med, Karolinska Institute, Stockholm, Sweden.

Correspondence: L. Didon, Dept of Genetic Medicine, Weill Cornell Medical College, 1300 York Ave, W401, New York, NY, USA. E-mail: lud2002@med.cornell.edu

Statement of Interest: None declared.

\section{REFERENCES}

1 Didon L, Roos AB, Elmberger GP, et al. Lung-specific inactivation of CCAAT/enhancer binding protein $\alpha$ causes a pathological pattern characteristic of COPD. Eur Respir J 2010; 35: 186-197.

2 Baraldi E, Filippone M. Chronic lung disease after premature birth. N Engl J Med 2007; 357: 1946-1955.

DOI: $10.1183 / 09031936.00024710$ 